\title{
Markers of hepatitis B infection and immunity in patients attending Aboriginal community controlled health services
}

\section{Mary E Harrod PhD \\ Sophia Couzos FRACGP, FACRRM, FAFPHM Associate Professor ${ }^{2}$ \\ Dea Delaney-Thiele
MPH Head, Population Health Research Unit ${ }^{3}$ \\ Gregory J Dore MPH, PhD, FRACP Head, Viral Hepatitis Clinical Research Program \\ Belinda Hammond Research Officer ${ }^{4}$ \\ Mark Saunders Policy Officer ${ }^{5}$ \\ Mary Belfrage MBBS, DRANZCOG, MRACGP Medical Director ${ }^{6}$ \\ Sidney Williams AdvDipMgt Research Officer \\ John M Kaldor Professor and Program Head of Public Health Interventions Research Group \\ James Ward \\ Deputy Head Aboriginal Health Program}

1 Kirby Institute, University of New South Wales, Sydney,

2 General Practice and Rural Medicine, James Cook University,

Townsville, QLD.

3 Public Health Unit, Aboriginal Medical Service Western Sydney, Sydney, NSW.

4 Quality, Safety and Reporting, Nunkuwarrin Yunti of South Australia Inc Adelaide, SA

5 National Aborigina

Community Controlled Health Organisation, Canberra, ACT.

6 Victorian Aboriginal Health

Service, Melbourne, VIC

7 Goondir Health Services, Dalby, QLD.

8 Baker IDI Central Australia, Alice Springs, NT.

mharrod@

kirby.unsw.edu.au

MJA 2014; 201: 339-342 doi: 10.5694/mjal4.00121

\section{$-$} epatitis $B$ virus (HBV) is an important cause of illness and death in Australia, with up to 218000 Australians living with chronic infection. ${ }^{1}$ Aboriginal and Torres Strait Islander people - with rates of chronic infection of $3.7 \%$ nationally compared with $0.9 \%$ in the general population ${ }^{2}$ and higher prevalence in remote communities - are recognised as a priority population in Australia's first National Hepatitis B Strategy, endorsed in 2010. ${ }^{3}$ Aboriginal and Torres Strait Islander people experience a rate of liver cancer up to 10 times that of the non-Indigenous population ${ }^{4}$ with an overall two-to-threefold increase in the rate of HBV-related liver cancer expected by 2017. ${ }^{3}$ Although a highly effective vaccine is now available and has been offered universally for newborns since 2000, many of those born before universal vaccination remain susceptible, and are at risk of exposure, primarily through sexual contact, including young adults not reached by adolescent catch-up programs. ${ }^{5-8}$ While the likelihood of acquiring chronic hepatitis B infection and experiencing long-term complications is far greater when exposure takes place in infancy, exposed adolescents and adults can experience serious acute illness, with a proportion leading to chronicity. ${ }^{9}$

An important strategy for the control of $\mathrm{HBV}$ is targeted testing of people at risk. Recent guidelines recommend that people at risk, including Aboriginal and Torres Strait Islander people, be offered hepatitis B surface antibody (HBsAb), hepatitis B surface antigen (HBsAg), and hepatitis B core antibody $(\mathrm{HBc} \mathrm{Ab})$ testing. The exception is in pregnancy, where testing for HBsAg only is recommended to detect chronic infection. Screening for all three markers concurrently allows a distinction to be made between the presence of chronic $\mathrm{HBV}$, immunity from vaccination or prior infection, and susceptibility to infection. ${ }^{10,11}$

Objective: Hepatitis B virus (HBV) infection remains an important cause of morbidity and mortality in Aboriginal and Torres Strait Islander people, who have high rates of infection compared with non-Indigenous Australians. We aimed to increase the evidence base around HBV in Aboriginal and Torres Strait Islander people through an analysis of routine clinical encounter data.

Design: A cross-sectional study of de-identified records from electronic patient systems over 5 years (8 January 2009 to 11 July 2013).

Setting: Four Aboriginal community controlled health services.

Participants: All patients attending for a clinical visit were included in the study. Hepatitis B testing records were included if at least one serological test for HBV was done.

Main outcome measures: Percentage of clinical patients tested for hepatitis B, compliance with guidelines and serological status.

Results: A total of 2959 people aged 15-54 years were screened for HBV, representing $17.2 \%$ of all people with a clinical visit in the study period. A total of 865 Aboriginal patients were tested concurrently for hepatitis B surface antigen (HBsAg), hepatitis B core antibody and hepatitis B surface antibody. Of those, $352(40.7 \%)$ were susceptible to HBV infection (95\% Cl, 37.4\%-43.9\%) and 34 (3.9\%) had either an acute or chronic infection indicated by a positive HBsAg result ( $95 \% \mathrm{Cl}, 2.6 \%-5.2 \%)$. In 329 women with antenatal screening, six (1.8\%) returned a positive HBsAg result ( $95 \% \mathrm{Cl}, 0.37 \%-3.28 \%)$.

Conclusion: A substantial proportion of patients tested were susceptible to $\mathrm{HBV}$, with a high percentage potentially infectious compared with the general population. High levels of active infection and susceptibility to infection suggest many opportunities for transmission and indicate the potential benefit of routine HBV testing and vaccination in this population.

In order to examine recent testing practice as well as the prevalence of markers of infection and susceptibility, we undertook a review of HBV serology testing patterns and positivity in patients attending four Aboriginal community controlled health services (ACCHS) - Nunkuwarrin Yunti South Australia Inc, Aboriginal Medical Service Western Sydney, the Victorian Aboriginal Health Service and Goondir Health Services. The study was aimed at supporting HBV prevention and management efforts in Aboriginal and Torres Strait Islander people receiving primary health care.

\section{Methods}

This study was a retrospective, crosssectional analysis of clinical encounter data contained within the medical records of patients attending four ACCHS. Services participated in the project through their involvement in REACCH (Research Excellence in Aboriginal Community-Controlled Health), a collaborative project funded by the National Health and Medical Research Council (NHMRC) and managed jointly by the Kirby Institute and the National Aboriginal Community Controlled Health Organisation. All services are providers of comprehensive primary health care to predominantly Aboriginal and Torres Strait Islander patients.

Data were collected from service patient information management systems through GRHANITE software which extracted specified variables in a de-identified, encrypted format. The accuracy of GRHANITE has been comprehensively assessed by internal reliability checks showing the software correctly classified $100 \%$ of pathology test results as positive or negative, and comparisons with external laboratory data, which showed $92 \%-95 \%$ concordance. ${ }^{12}$ Records of 
1 Serological profiles used to define hepatitis B virus (HBV) infection status*

\begin{tabular}{lccc} 
& \multicolumn{3}{c}{ HBV marker } \\
\cline { 2 - 4 } Serological profile & HBsAg & HBcAb & HBsAb \\
\hline Susceptible & Negative & Negative & Negative \\
Immune due to natural infection & Negative & Positive & Positive \\
Immune due to HBV vaccination & Negative & Negative & Positive \\
Active (acute or chronic) infection & Positive & Positive & Negative \\
Interpretation unclear & Negative & Positive & Negative \\
\hline
\end{tabular}

$\mathrm{HBCAb}=$ hepatitis B core antibody. $\mathrm{HBs} A \mathrm{~b}=$ hepatitis $\mathrm{B}$ surface antibody. $\mathrm{HBsAg}=$ hepatitis $\mathrm{B}$ surface antigen. *Based on the United States Centers for Disease Control and Prevention guidelines. ${ }^{13}$

patient visits were included if they related to a clinical visit of a person aged from 15 to 54 years from 8 January 2009 to 11 July 2013. For each patient record, information was extracted on age, sex, Aboriginal and Torres Strait Islander status and HBV testing if completed. HBV serology results were obtained from pathology test data and coded as positive or negative. The number of antenatal patients was derived from clients marked as antenatal in the patient system and with a corresponding visit record. The pattern of hepatitis serology testing was analysed by Aboriginal status, age and sex. Serology results were examined for patients with concurrent testing for $\mathrm{HBsAg}, \mathrm{HBsAb}$ and $\mathrm{HBcAb}$, and classified according to the United States Centers for Disease Control and Prevention guidelines (Box 1). ${ }^{13}$ For patients who had these three markers tested concurrently once, only the results of the most recent occasion of testing were included in the analysis. We conducted logistic regression and calculated odds ratios (ORs) examining the relationship between testing outcomes, sex and age. All statistical analyses were conducted using Stata, version 12 (StataCorp).

The study was reviewed and approved by the board of management of each participating service and was reviewed by the Aboriginal Health and Medical Research Council Ethics Committee (New South Wales) and the Aboriginal Health Research and Ethics Committee (South Australia) and endorsed by the University of New South Wales Human Research Ethics Committee.

\section{Results}

A total of 17180 patients aged $15-54$ years were recorded as having attended the participating clinics over the study period (8 January 2009 to 11 July 2013), with 2959 people (17.2\%) serologically tested for HBV in total over 3832 occasions of testing. Of the patients attending, 11872 (69.1\%) were Aboriginal and Torres Strait Islander (Box 2), and of these, 2435 (20.5\%) were tested for HBV on 3221 occasions. Of the 3832 occasions of HBV testing in the study period, 1121 (29.3\%) involved concurrent testing for $\mathrm{HBsAg}$, $\mathrm{HBsAb}$ and $\mathrm{HBcAb}$ in 995 individuals. A total of 983 occasions of concurrent testing occurred in 865 Aboriginal and Torres Strait Islander patients. Further results relate to this
2 Aboriginal and Torres Strait Islander clients seen at participating clinics, and proportions serologically tested for any hepatitis B virus infection marker by sex, age group and antenatal status

\begin{tabular}{|c|c|c|c|c|c|c|c|c|c|}
\hline \multirow[b]{2}{*}{ Clients } & \multicolumn{8}{|c|}{ Age group (years) } & \multirow[b]{2}{*}{ Totals } \\
\hline & $15-19$ & $20-24$ & $25-29$ & $30-34$ & $35-39$ & 40-44 & $45-49$ & $50-54$ & \\
\hline \multicolumn{10}{|l|}{ Women } \\
\hline Clients $(n)$ & 818 & 1090 & 905 & 864 & 794 & 810 & 679 & 705 & 6665 \\
\hline$\%$ tested & $29.1 \%$ & $28.0 \%$ & $27.4 \%$ & $25.7 \%$ & $22.8 \%$ & $15.6 \%$ & $14.0 \%$ & $11.1 \%$ & $22.4 \%$ \\
\hline$\%$ antenatal & $18.3 \%$ & $14.7 \%$ & $8.7 \%$ & $6.4 \%$ & $3.0 \%$ & $0.7 \%$ & 0 & 0 & $7.1 \%$ \\
\hline \multicolumn{10}{|l|}{ Men } \\
\hline Clients $(n)$ & 685 & 745 & 680 & 601 & 654 & 684 & 600 & 558 & 5207 \\
\hline$\%$ tested & $9.6 \%$ & $20.8 \%$ & $17.1 \%$ & $19.5 \%$ & $24.0 \%$ & $18.3 \%$ & $22.2 \%$ & $13.1 \%$ & $18.1 \%$ \\
\hline
\end{tabular}

subgroup only. Of these 865 patients with complete testing, 352 ( $40.7 \%$; $95 \%$ CI, $37.4 \%-43.9 \%$ ) were classified as susceptible to HBV infection, 115 (13.3\%; 95\% CI, $11.0 \%-15.6 \%)$ were immune due to natural infection, 319 (36.9\%; 95\% CI, 33.7\%-40.1\%) were immune due to vaccination, 34 (3.9\%; 95\% CI, 2.6\%-5.2\%) had either an acute or chronic infection and 45 (5.2\%; $95 \%$ CI, 3.7\%-6.7\%) had an unclear result (Box 3). When examined by age and sex (Box 4), patients aged 30-54 years were less likely to be immune due to vaccination than those aged $15-29$ years $(\mathrm{OR}, 0.36$; $95 \% \mathrm{CI}$, $0.28-0.49)$, and men were more likely to be vaccinated than women (OR, $1.38 ; 95 \%$ CI, 1.04-1.83). Patients aged 30-54 years were more likely than those aged 15-29 years to be previously exposed to HBV and either immune through natural infection (OR, 8.67; 95\% CI, 4.9-15.4) or HBsAg positive indicating active or chronic infection (OR, 2.45; 95\% CI, 1.2-5.9). A lack of antibodies to HBV, through either vaccination or prior infection, was not related to age or sex.

A total of 572 women aged between 15 and 48 years seen at the services were pregnant, including 474 Aboriginal and Torres Strait Islander women aged 15-44 years, 329 of whom $(69.4 \%)$ were tested for HBsAg with six positive results; a prevalence of $1.8 \%$ (95\% CI, $0.37 \%-3.28 \%$ ).

\section{Discussion}

The analyses reported are of a substantial sample of Aboriginal and Torres Strait Islander people tested for all three hepatitis B serological markers drawn from urban and regional ACCHS, and are the first data of their kind available in this setting. In this study, about $40 \%$ of Aboriginal adolescents and adults remain susceptible to HBV infection. Potential infectiousness indicated by a positive HBsAg result was high at nearly $4 \%$, but with significant variation by age (Box 3 and Box 4). The high levels of active infection and susceptibility to HBV infection suggest many opportunities for transmission, primarily through sexual partnerships but also in close household contacts.

These results may not be representative of the clinic population as 
whole, or the broader Aboriginal and Torres Strait Islander population in the areas served by these clinics, as this only represents a subset of clinic patients. The high rates of acute or chronic HBV infection may reflect selective testing of patients. In this case, the true prevalence of chronic infection in this patient population could be lower than the $3.9 \%$ reported. The proportion of positive HBsAg tests of $1.8 \%$ in antenatal women may be a more accurate indicator of infection in younger patients.

It is also important to note that a negative $\mathrm{HBs} \mathrm{Ab}$ result, as defined by a titre of $<10 \mathrm{mIU} / \mathrm{mL}$, does not necessarily indicate the absence of vaccination, as titres decline to below this level in up to $50 \%$ of people receiving a full course of vaccination after less than a decade. ${ }^{14,15}$ Protection appears to be maintained for at least two decades after vaccination in healthy individuals who achieved an initial response to vaccine. ${ }^{14}$ Therefore, a proportion of the study sample defined serologically as "susceptible" may have been vaccinated.

Understanding HBV population prevalence can guide clinical service policy and practice regarding $\mathrm{HBV}$ testing, immunisation and health promotion. The retrospective data reported here indicate that a quarter of the patients who had some form of HBV testing had testing in accordance with recent guidelines, a high proportion when compared with

3 Testing outcomes by age group and sex for 865 Aboriginal and Torres Strait Islander patients who were tested concurrently for hepatitis B surface antibody, hepatitis B surface antigen and hepatitis B core antibody*

\begin{tabular}{|c|c|c|c|c|c|}
\hline \multirow[b]{2}{*}{ Testing outcome } & \multicolumn{2}{|c|}{ Women } & \multicolumn{2}{|c|}{ Men } & \multirow[b]{2}{*}{ Total } \\
\hline & $15-29$ years & $30+$ years & $15-29$ years & $30+$ years & \\
\hline Susceptible & $117(46.1 \%)$ & $104(40.5 \%)$ & $47(37 \%)$ & $84(37 \%)$ & $352(40.7 \%)$ \\
\hline Immune due to natural infection & $9(3.5 \%)$ & $58(22.6 \%)$ & $2(1.6 \%)$ & $46(20.3 \%)$ & 115 (13.3\%) \\
\hline Immune due to vaccination & 119 (46.9\%) & $66(25.7 \%)$ & $72(56.7 \%)$ & $62(27.3 \%)$ & $319(36.9 \%)$ \\
\hline Acute or chronic infection & $6(2.4 \%)$ & $10(3.9 \%)$ & $1(0.8 \%)$ & $17(7.5 \%)$ & $34(3.9 \%)$ \\
\hline Unclear & $3(1.2 \%)$ & $19(7.4 \%)$ & $5(3.9 \%)$ & $18(7.9 \%)$ & $45(5.2 \%)$ \\
\hline Total tested $(n)$ & 254 & 257 & 127 & 227 & 865 \\
\hline
\end{tabular}

* Results classified in accordance with the United States Centers for Disease Control and Prevention guidelines. ${ }^{13}$

previous reports in primary care ${ }^{16}$ and one that is likely to increase as the guidelines become more widely adopted in clinical practice.

Australian vaccination guidelines recommend Aboriginal Torres Strait Islander people be offered testing for previous HBV infection and vaccination if non-immune. ${ }^{17}$ There are jurisdictional inconsistencies in funding HBV vaccination in adult Aboriginal and Torres Strait Islander people who are found to be non-immune after testing, which may reflect the heterogeneity of risk on the basis of ethnicity criteria alone. ${ }^{18-25}$ Increased focus on appropriate testing and a nationally consistent approach to vaccination that is designed to provide optimal benefit for Aboriginal and Torres Strait Islander people at risk would assist in further reducing the burden of HBV infection in

4 Prevalence of hepatitis $B$ virus testing outcomes by age group and sex for 865 Aboriginal and Torres Strait Islander patients tested for HBsAg, HBsAb and HBcAb concurrently

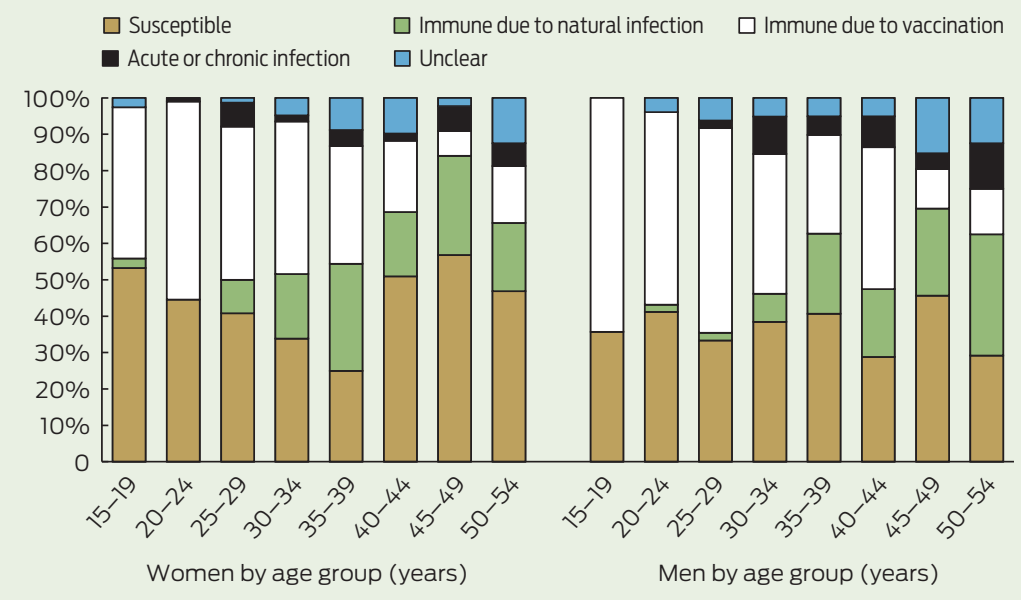

$\mathrm{HBCAb}=$ hepatitis $\mathrm{B}$ core antibody. $\mathrm{HBsAb}=$ hepatitis B surface antibody. $\mathrm{HBsAg}=$ hepatitis B surface antigen. a population that has been substantially affected by its consequences.

Acknowledgements: REACCH is a Centre for Research Excellence funded by the NHMRC.

Competing interests: No relevant disclosures.

Received 27 Jan 2014, accepted 21 May 2014.

1 MacLachlan JH, Allard N, Towell V, Cowie BC. The burden of chronic hepatitis $B$ virus infection in Australia, 2011. Aust N Z J Public Health 2013; 37: 416-422.

2 Graham S, Guy RJ, Cowie B, et al. Chronic hepatitis $B$ prevalence among Aboriginal and Torres Strait Islander Australians since universal vaccination: a systematic review and meta-analysis. BMC Infect Dis 2013; 13: 403.

3 Australian Government Department of Health and Ageing. National Hepatitis B Strategy, 2010-2013. Canberra: Commonwealth of Australia, 2010. http://www.health.gov.au/ internet/main/publishing.nsf/Content/ohpnational-strategies-2010-hepb/\$File/hepb.pdf (accessed May 2014)

4 Condon JR, Armstong BK, Barnes A, Cunningham J. Cancer in Indigenous Australians: a review. Cancer Causes Control 2003; 14: 109-121.

5 Gidding HF, Warlow M, Maclntyre CR, et al. The impact of a new universal infant and schoolbased adolescent hepatitis $B$ vaccination program in Australia. Vaccine 2007; 25 8637-8641.

6 Cowie B, Karapanagiotidis T, Enriquez A, Kelly $H$. Markers of hepatitis B virus infection and immunity in Victoria, Australia, 1995 to 2005. Aust N Z J Public Health 2010; 34: 72-78.

7 Skinner R, Nolan T. Adolescent hepatitis B immunisation -- should it be the law? Aust NZ J Public Health 2001; 25: 230-233.

8 Skinner SR, Imberger A, Nolan T, et al. Randomised controlled trial of an educational strategy to increase school-based adolescent hepatitis B vaccination. Aust N Z J Public Health 2000; 24: 298-304.

9 Lavanchy D. Hepatitis B virus epidemiology, disease burden, treatment, and current and emerging prevention and control measures. J Viral Hepat 2004; 11: 97-107.

10 National Aboriginal Community Controlled Health Organisation, Royal Australian College of General Practitioners. National guide to a preventive health assessment for Aboriginal and Torres Strait Islander people. 2nd ed. Melbourne: RAGCP, 2012. http://www.racgp. org.au/your-practice/guidelines/nationalguide (accessed May 2014).

11 Department of Health. National hepatitis B testing policy. Canberra: Commonwealth of 
Australia, 2012. http://testingportal.ashm.org au/hbv (accessed May 2014).

12 Boyle D, Kong F. A systematic mechanism for the ethical collection and interpretation of display format pathology test results from Australian primary care records. Electronic Journal of Health Informatics [internet] 2011; 6: el8.

13 US Department of Health and Human Services, Centers for Disease Control and Prevention. Interpretation of hepatitis B serologic test results. http://www.cdc.gov/hepatitis/hbv/ pdfs/serologicchartv8.pdf (accessed Jan 2013).

14 Jack AD, Hall AJ, Maine N, et al. What level of hepatitis B antibody is protective? J Infect Dis 1999; 179: 489-492.

15 Leuridan E, Van Damme P. Hepatitis B and the need for a booster dose. Clin Infect Dis 2011; 53 . 68-75.

16 Carroll E, Davis JS. Incomplete hepatitis B screening prevents an adequate public health response in Aboriginal communities. Australian Indigenous Health Bulletin [internet] 2010; 10. http://healthbulletin.org.au/articles/ incomplete-hepatitis-b-screening-prevents- an-adequate-public-health-response-inaboriginal-communities (accessed May 2014).

17 Australian Government Department of Health. The Australian immunisation handbook. 10th ed. Canberra: Australian Government, 2013.

18 Victorian Government Department of Health. Immunisation schedule Victoria. 2013. http:// www.health.vic.gov.au/immunisation/ factsheets/schedule-victoria.htm (accessed May 2014).

19 NSW Government Ministry of Health. Hepatitis B vaccination policy. 2005. http://www0. health.nsw.gov.au/policies/pd/2005/pdf/ PD2005_222.pdf (accessed May 2014).

20 Queensland Government. Hepatitis B. http://conditions.health.qld.gov.au/ HealthConditions/6/Child-Health/154/ Immunisation/848/Hepatitis-B (accessed Sep 2014).

21 Government of South Australia, SA Health. High risk hepatitis B immunisation program. http://www.sahealth.sa.gov.au/wps/wcm/ connect/public+content/sa+health+internet/ health+topics/health+conditions+prev ention+and+treatment/immunisation/ immunisation+programs/high+risk+hepatitis+ b+immunisation+program (accessed Apr 2014).

22 ACT Government Health. ACT immunisation strategy 2012-2016. http://health.act.gov. au/c/health?a=dlpubpoldoc\&document $=2802$ (accessed Apr 2014).

23 Government of Western Australia, Department of Health, Communicable Disease Control Directorate. Hepatitis B vaccination program [operational directive]. 27 Nov 2009. http:// www.health.wa.gov.au/circularsnew/ pdfs/12576.pdf (accessed May 2014).

24 National Immunisation Program - Tasmania. Aboriginal and Torres Strait Islander adult and child immunisation schedule. 2013. http://www.dhhs.tas.gov.au/ data/assets/ pdf_file/0011/88760/Adult_and_Child_ Aboriginal_and_Torres_Strait_Islander Immunisation_Schedule_July_2013.pdf (accessed Apr 2014).

25 Northern Territory Government. Adult and special groups vaccination schedule. 2013. http://www.health.nt.gov.au/library/ scripts/objectifyMedia.aspx?file=pdf/63/90 pdf\&sitelD=1\&str_title=Adult $\% 20$ and $\% 20$ Special\%20Groups\%20Vaccination\%20 Schedule.pdf (accessed Apr 2014). 